\title{
Gauge Field, Fermi-Bose Duality, Minimization Entropy Reduction and Degeneracy of Critical Temperatures
}

\author{
Yougang Feng \\ College of Physics, Guizhou University, Guiyang, China \\ Email: ygfeng45@aliyun.com
}

How to cite this paper: Feng, Y.G. (2019) Gauge Field, Fermi-Bose Duality, Minimization Entropy Reduction and Degeneracy of Critical Temperatures. Journal of Modern Physics, 10, 1149-1156.

https://doi.org/10.4236/jmp.2019.109075

Received: July 30, 2019

Accepted: August 11, 2019

Published: August 14, 2019

Copyright (อ 2019 by author(s) and Scientific Research Publishing Inc. This work is licensed under the Creative Commons Attribution International License (CC BY 4.0).

http://creativecommons.org/licenses/by/4.0/

\section{c) (i) Open Access}

\begin{abstract}
A non-Abelian gauge field is introduced to maintain the gauge invariance for the self-similar transformation, resulting in three conservation laws. It has been found that the non-Abelian gauge field is suitable to electromagnetic interaction. The Fermi-Bose duality for blocks makes blocks act as both fermi-blocks involving in spin interaction and non-spin interaction and bose-blocks carrying non-spin interaction. The self-similar transformation requires the block type inversion guaranteed by the gauge field. There is a constant and minimization entropy reduction in the transformation. It is realized that there is degeneracy of critical temperatures in the experiments for real materials. The thermodynamic dark parameters associated with the non-spin interaction should be taken seriously.
\end{abstract}

\section{Keywords}

Gauge, Fermi-Bose Duality, Inversion, Entropy, Dark Parameters,

Degeneracy

\section{Introduction}

Quantum field theory has been applied in the research on critical phenomena, for example, by means of renormalization group theory, perturbation theory, and Feynman graphs to compute critical points and critical exponents [1] [2] [3]. The renormalization group itself is a symmetry group, in which transformation we found coexistence of ordered blocks and disordered blocks. On this basis, the concept of isomorph spin is set up, and then some hidden symmetries have been revealed [4]. The two types of blocks make us recall the coexistence of protons and neutrons, and further, we think that there must be a gauge field to maintain 
the transformation. According to the definition put forward in reference [4], the $n_{+}$-blocks are ordered blocks, with which coexisting the $n_{-}$-blocks are disordered. Each lattice point is similar to a fermion, and they can form an $n_{+}$-block spin behaving as a fermion. We call the $n_{+}$-block as fermi-block. An $n_{+}$-block spin has $Z$ (coordinate number) nearest neighbor $n_{+}$-blocks, and these $Z$ coupled block spins have different energy states due to Pauli exclusion principle, leading to that this block spin has $Z$ different spin states at least. This is the reason why we yet introduce hyperspin $Y_{f}$ for the blocks, besides the isomorph spin $I_{f}$ [4]. Furthermore, a block is a set of lattices; it is these lattices that give the block a peculiar ability to possess more states a particle not having. For example, two states of $I_{f}=1 / 2$ and $I_{f}=1$ [4], by which a block can behave as either fermi-block participating in spin interaction and non-spin interaction or bose-block carrying the non-spin interaction, which will be illustrated in Section 2.

The spin interaction is essentially electromagnetic interaction in which spins take part. Since each block contains charged lattice spins, the block always has nonzero charge although it may have zero-spin. The so-called non-spin interaction is different from neither weak interaction nor strong interaction; it is just an electromagnetic interaction in which no spin involve. Particle physics tells us that the electromagnetic field is, in nature, an Abelian gauge field introduced to maintain the invariance $\mathrm{U}(1)$ of electromagnetic interaction. Non-Abelian gauge field is available for those interactions to keep the invariance of $S U(2)$, etc. [5]. Studying continuous phase transition, however, we see that there is localized $\mathrm{SU}(2)$ symmetry describing non-spin electromagnetic interaction, indicating that non-Abelian gauge field is also suitable for electromagnetic interaction. For this reason, we neglect mass to explore the gauge field in this paper.

In Section 2, at first, we introduce a gauge field on a finite hierarchy of the self-similar transformation at temperature $T$, and get $W_{+}$-blocks, $W_{-}$-blocks, and $Z_{0}$-blocks carrying the non-spin interaction like bosons. We call them as bose-blocks. Then, we discover Fermi-Bose duality for blocks, we've revised the explanation of the excitation states and the intermediate states of reference [4]. In Section 3, we discuss gauge field relating to the block type inversion when temperature charges and system entropy evolution, finding constant $k_{B} \ln 2$ and minimization entropy reduction. We explain why the scaling law only occurs nearby the first critical temperature $T_{c f}$ and there is degeneracy of critical temperatures for experimental specimens; point out that the thermodynamic dark parameters will play important roles in the further research on phase transition. Section 4 is conclusion remark.

\section{Theory}

\subsection{Gauge Field and Three Conservation Laws}

On the $r$-th hierarchy, $0<r<+\infty$, at temperature $T_{m}$, the state of an ordered $n_{+}^{m}$-block is $\psi_{+}^{m}$ (the superscript $m$ relates to $T_{m}$, the following are the same as), the state of a disordered $n_{-}^{m}$-block is $\psi_{0}^{m}$, the two states are regarded as iso- 
morph spin doublet for an identical block, represented as

$$
\psi=\left(\begin{array}{c}
\psi_{0}^{m} \\
\psi_{+}^{m}
\end{array}\right)
$$

its conjugate state is $\bar{\psi}$. The Lagrangian density of the interaction in which a gauge field participates is expressed by [6]

$$
L^{m}=\bar{\psi} i \gamma^{\mu}\left(\partial_{\mu}-i g T^{i} A_{\mu}^{i}\right) \psi-\frac{1}{4} F_{\mu \nu}^{i} F^{i \mu v}
$$

The kinetic energy term for the non-Abelian field is $(-1 / 4) F_{\mu \nu}^{i} F^{i \mu v}$, and

$$
F_{\mu \nu}^{i}=\partial_{\mu} A_{v}^{i}-\partial_{v} A_{\mu}^{i}+g \varepsilon^{i j k} A_{\mu}^{j} A_{v}^{k}
$$

where $g$ is a constant. The term of interaction between the gauge field and the isomorph spin field is

$$
g \bar{\psi} \gamma^{\mu} T^{i} A_{\mu}^{i} \psi
$$

There are three matrices for $T^{i}, i=1,2,3$,

$$
T^{1}=\frac{1}{2}\left(\begin{array}{ll}
0 & 1 \\
1 & 0
\end{array}\right), T^{2}=\frac{1}{2}\left(\begin{array}{cc}
0 & -i \\
i & 0
\end{array}\right), T^{3}=\frac{1}{2}\left(\begin{array}{cc}
1 & 0 \\
0 & -1
\end{array}\right)
$$

Substituting Formula (5) into Formula (4), we get

$$
\frac{1}{2} g \bar{\psi} \gamma^{\mu}\left(\begin{array}{cc}
Z_{0}^{m} & \sqrt{2} W_{-}^{m} \\
\sqrt{2} W_{+}^{m} & -Z_{0}^{m}
\end{array}\right) \psi
$$

$W_{+}^{m}$ and $W_{-}^{m}$ are newly defined fields:

$$
W_{-}^{m}=(1 / \sqrt{2})\left(A_{\mu}^{1}-i A_{\mu}^{2}\right), \quad W_{+}^{m}=(1 / \sqrt{2})\left(A_{\mu}^{1}+i A_{\mu}^{2}\right)
$$

And the third new field follows

$$
Z_{0}^{m}=A_{\mu}^{3}
$$

The definition of $W_{-}^{m}$ and $W_{+}^{m}$ differs from that of the particle physics [5]. Using Formula (1), the Formula (6) becomes

$$
\frac{g}{2}\left[\overline{\psi_{0}^{m}} \gamma^{\mu} Z_{0}^{m} \psi_{0}^{m}+\sqrt{2} \overline{\psi_{0}^{m}} \gamma^{\mu} W_{-, \mu}^{m} \psi_{+}^{m}+\sqrt{2} \overline{\psi_{+}^{m}} \gamma^{\mu} W_{+, \mu}^{m} \psi_{0}^{m}-\overline{\psi_{+}^{m}} \gamma^{\mu} Z_{0}^{m} \psi_{+}^{m}\right]
$$

Each term contains three block states, $i=1,2$, 3, which parameters satisfy three conservation laws:

$$
\sum_{i=1}^{3} Y_{f, i}=0, \quad \sum_{i=1}^{3} S_{i}=0, \quad \sum_{i=1}^{3} I_{f 3, i}=0
$$

where the units for $Y_{f} S$, and $I_{f}$ are defined by reference [4]. The states $W_{+}^{m}$, $W_{-}^{m}$, and $Z_{0}^{m}$ correspond to $W_{+}^{m}$-blocks, $W_{-}^{m}$-blocks, and $Z_{0}^{m}$-blocks, respectively, carrying the non-spin interaction, and they are bose-blocks. The bose-blocks are somewhat like the Cooper electron pairs of BCS theory of superconductivity [7]. In addition, the four terms of Formula (9) show there are four kinds of the non-spin interactions and seems to be no limit to the interaction length, unlike that the spin interaction is limited to blocks as their neighbors (Ising models). 


\subsection{Fermi-Bose Duality}

Referring to Figure 1 and Figure 3 of reference [4], the structure of a $n_{+}$-block for the plane square lattice system is different from that of a $n_{+}$-block for a plane triangle lattice system. For the latter, a $n_{+}$-block and a $n_{-}$-block are clearly separated. For the former, a $n_{+}$-block consists of $n_{+}$lattices, $n_{-}$of which construct a $n_{-}$-block, $n_{-}<n_{+}$. According to the renormalization group theory, when we only consider a block its inner structure is indistinguishable, namely, the lattices can be neglected. For the plane square lattice system, as the symmetry center of a $n_{+}$-block and the symmetry center of a $n_{-}$-block are not superposed, so they are two blocks, just as the center of positive charge and the center of negative charge of a water molecule of three atoms containing positive charges and negative charges are not covered with each other, and the water molecule is a polar molecule, the two charges hence play their respective roles. The above interpretation is also applicable to the simply cubic lattice system.

Referring to Figure 5 of reference [4], the following symbol $\rightarrow$ represents corresponding relation between states, with the relevant parameters listed:

$$
\begin{gathered}
\psi_{+}^{m} \rightarrow A_{+}: Y_{f}=1, S=1, I_{f 3}=1 / 2 ; \\
\psi_{0}^{m} \rightarrow B_{+}: Y_{f}=1, S=0, I_{f 3}=-1 / 2 ; \\
\overline{\psi_{+}^{m}} \rightarrow A_{-}: Y_{f}=-1, S=-1, I_{f 3}=-1 / 2 ; \\
\overline{\psi_{0}^{m}} \rightarrow B_{-}: Y_{f}=-1, S=0, I_{f 3}=1 / 2 .
\end{gathered}
$$

As there is not any other type of block except the $n_{+}$-blocks and the $n_{-}$-blocks, the Fermi-Bose duality makes the existences of $W_{+}$-blocks, $W_{-}$-blocks, and $Z_{0}$-blocks become possible:

$$
\begin{gathered}
W_{+}^{m} \rightarrow A_{+}^{*}: Y_{f}=0, S=1, I_{f 3}=1 ; \\
W_{-}^{m} \rightarrow A_{-}^{*}: Y_{f}=0, S=-1, I_{f 3}=-1 ; \\
Z_{0}^{m} \rightarrow A_{0} \text { or } B_{0}: Y_{f}=0, S=0, I_{f 3}=0 .
\end{gathered}
$$

The $A_{+}$and $A_{-}$are the ground states for the $n_{+}$-blocks, the $B_{+}$and $B_{-}$are the ground states for the $n_{-}$-blocks, they have the same isomorph spin $I_{f}=1 / 2$. The $A_{+}^{*}$ and $A_{-}^{*}$ are the excitation states for the $n_{+}$-blocks, with $I_{f}=1$, for which the explanation of reference [4] is not precise. As the same reason, the intermediate states $A_{0}$ and $B_{0}$ come out of the $n_{+}$-blocks and the $n_{-}$-blocks, respectively. All these states are originated in the $n_{+}$-blocks and the $n_{-}$-blocks, belonging to the isomorph spin octet. By the previous corresponding relations, for example, the parameters of the states $B_{-}, A_{-}^{*}$, and $A_{+}$in the second term of Formula (9) successively are $Y_{f, 1}=-1, S_{1}=0, I_{f 3,1}=1 / 2, \quad Y_{f, 2}=0, S_{2}=-1$, $I_{f 3,2}=-1, \quad Y_{f, 3}=1, \quad S_{3}=1, \quad I_{f 3,3}=1 / 2$, accord with the conservation laws given by Formula (10).

An important feature is that the formula can only describe the initial or final states of the interaction, and cannot show the intermediate process. Actually, there is not any block having a changeless state, every block is in a dynamic 
process, all the states are changing, at the same time comply with the relative symmetry and the conservation laws. As geometric positions cannot be changed the non-spin interaction only is accomplished by exchanging states. Let states $\alpha$, $\beta$, and $\lambda$ are in the same term, $\alpha$ and $\beta$ interact through $\lambda$, one possible way to do this is as follows: The $\alpha$ goes to the $\lambda$, the $\lambda$ goes to the $\alpha$ in turn; then the $\beta$ goes to the $\lambda$, and inversely, the $\lambda$ goes to the $\beta$; by swapping with the $\lambda$, the original $\alpha$ and $\beta$ exchange. If you continue like this, you may revert to your original state. This picture is available for the first and fourth terms of Formula (9). Another scene may be suitable to the interactions in the second and third terms of the formula: As all state transition, excitation, and degeneration should go through the intermediate states, the exchange with the excited state is completed in there. What's more, the intermediate states of the $n_{+}$-blocks and the $n_{-}$-blocks are exactly the same, which is the property that allows the interactions to come true. Meanwhile, there could be a situation where the original states in the second term are interchanged with the original states in the third term by one-to-one correspondence way, keeping the Lagrangian density constant. Balance the energy must be in the interactions.

We can think of those states in each interaction term as bound states made up of blocks. The three conservation laws list a restricted condition for the block spin states in the non-spin interaction, embodied in the fourth term of Formula (9): The block spin parallel pairs are forbidden to participate in this interaction. It shows that the block spin states are, in the non-spin interaction sense, not randomly distributed, but a conditional combination.

On the other hand, both combinations of spin parallel and spin anti-parallel are allowed in the spin interaction. Considering the two kinds of interactions, it can be seen that the way of the spin anti-parallel has larger existence probability, such that the total spin is always zero at any finite hierarchy of the transformation course, and the system becomes ordered only if through infinitely hierarchical transformation. As the spin-up parallel and the spin-down parallel are two states of energy lower than that of the anti-parallel state, they are two sides for the block magnetic spin-pair energy symmetry, having the same value, which evidences there is no Goldstone bosons derived from the spontaneous symmetry [8] [9].

\section{Discussion}

\subsection{Block Type Inversion}

Temperatures $T_{m+1}$ and $T_{m}$ are, $T_{c s}<T_{m}<T_{m+1} \leq T_{c f}$, so close to each other that the $n_{+}^{m+1}$-blocks transfer into the $n_{-}^{m}$-blocks when temperature changes from $T_{m+1}$ into $T_{m}$. Because the block's fractal dimension is a monotonically increasing function of the block side length in the region $T_{c s}<T \leq T_{c f}$, the $T_{c s}$ is the secondary phase transition critical temperature [4]. The longer the side length is, the larger the dimension will be, when the temperature drops [9]. This means that there is block type inversion. For example, in the plane triangle lattice spin system: 
$n_{-}^{m+1}=19, n_{+}^{m+1}=20$, and $n_{-}^{m}=20, n_{+}^{m}=21$. The ordered $n_{+}^{m+1}$-block at $T_{m+1}$ turns into the disordered $n_{-}^{m}$-block at $T_{m}$. If the $n_{+}^{m+1}$-block kept the state $\overline{\psi_{+}^{m+1}}$, instead of the state $\overline{\psi_{0}^{m}}$, the second interaction term in Formula (9) would show as

$$
\sqrt{2} \overline{\psi_{+}^{m+1}} \gamma^{\mu} W_{-, \mu}^{m} \psi_{+}^{m}
$$

This term violates the conservation laws of Formula (10), so it won't exist. Apparently, it is the gauge field that forces the $n_{+}^{m+1}$-blocks to reverse into the $n_{-}^{m}$ -blocks, keeping the gauge invariance.

\subsection{Constant $k_{B} \ln 2$ and Minimization Entropy Reduction}

Because of the additive nature of entropy, we divide the system entropy $H_{e}$ relating to magnetism into two parts: The first part $H_{e 1}$ is made by the existence probabilities of the $n_{+}$-blocks and the $n_{-}$-blocks, and the second, $H_{e 2}$, is determined by the various states combinations of these two types of blocks. The first part is denoted as $H_{e 1}[10]$,

$$
H_{e 1}=-k_{B}\left[P_{+} \ln P_{+}+P_{-} \ln P_{-}\right]
$$

where $k_{B}$ is Boltzmann constant, $P_{+}$and $P_{-}$are the occurrence probabilities for $n_{+}$-blocks and $n_{-}$-blocks, respectively. As $P_{+}=P_{-}=1 / 2$, we get

$$
H_{e 1}=k_{B} \ln 2
$$

The constant is available for the region $T_{c s}<T \leq T_{c f}$. The second part is constant independent of the finite hierarchy since the symmetry of the self-similar transformation. Therefore, the self-similar transformation for a certain temperature is an isentropic process.

On one hand, the magnitude of the system entropy loss is $\left|\Delta H_{e,+}^{m}\right|, \Delta H_{e,+}^{m}<0$, caused by the $n_{+}^{m}$-blocks for the degree of order of the system increases with the temperature decreases. On the other hand, the $n_{-}^{m}$-blocks are the disordered blocks closest to the $n_{+}^{m}$-blocks at $T_{m}$, responsible for the system entropy increase $\Delta H_{e,-}^{m}>0$, so the entropy reduction is minimized. The change in the system entropy takes

$$
\Delta H_{e}^{m}=\Delta H_{e,+}^{m}+\Delta H_{e,-}^{m}<0
$$

We understand that for a certain temperature $T_{m}$, the $n_{+}^{m}$-block is as small as possible ordered block, the $n_{-}^{m}$-block is as large as possible disordered block, this is an ordered path to minimum entropy reduction.

As $T_{m}$ is an arbitrary temperature in the previous temperature region, we have

$$
\mathrm{d}^{2} H_{e} / \mathrm{d} T^{2} \approx 0
$$

The decrease in the system entropy with the temperature declining is approximately homogeneous.

\subsection{Degeneracy of Critical Temperatures}

The lattice spin correlation length is the same as the block side length, depend- 
ing on temperature. The lower the temperature is, the larger both lengths become. The temperature at which all transition hierarchies disappear is defined as the secondary phase transition critical temperature $T_{c s}$. The largest lengths occur at the $T_{c s}$. The lengths corresponding to the block minimum fractal dimension appear at the $T_{c f}$ [4]. In addition, the system's size restricts the values of both the largest side length and the correlation length. For an infinite system, if the block side length is finite in the transformation course, the hierarchical number is infinite. When the whole system becomes a block with its side length equaling the system's size, the hierarchy vanishes; while both lengths are infinite. There is a large enough temperature gap between $T_{c s}$ and $T_{c f}$ which we call the renormalization region, where the scaling law exists [9]. For a finite system, if the side length is smaller than the system's size in the changing course, the number of the hierarchies are finite. When the side length turns into the system's size, the whole system is an isolated block and the hierarchy vanishes, while the correlation length is the same as the system's size.

The object of our theoretical study is an infinite system, the predicted laws cannot be fully displayed in the actual samples. All experimental specimens have only very limited size and one feature in common: the scaling law always appears nearby $T_{c f}$ It is the very finite size that leads to few transformation hierarchies. The $T_{c s}$ is so close to the $T_{c f}$ that the experimenters cannot distinguish both of them. The false image misleads to that there were no concepts of the $T_{c s}$ and the secondary phase transition. An important sign of the indistinguishability of the two critical temperatures is that the correlation length nearby $T_{c f}$ has covered entire specimens. We call this phenomenon as degeneracy of critical temperatures. Actual materials having the same molecular formula and crystal structure commonly share the same inherent $T_{c f}$ If they are all small, they all have the degeneracy phenomenon, otherwise the degeneracy will disappear: the larger the size, the lower the $T_{c s}$.

The analogous case concerns the compute simulation experiments [11] [12]. It's said that there is no block soon after the first phase transition nearby $T_{c f}$ In fact, the whole system has, at that moment, become an isolated block with its side length comparable to the system's size for the system is quite limited. One final point to note is that since we are concern about the phase transition under thermodynamic equilibrium state the simulation should insist the critical limit principle of maximum entropy and take the ordered path to minimum entropy reduction [9]. We expect the simulation test for a massive system on giant supercomputers may eliminate the degeneracy phenomenon.

\subsection{Thermodynamic Dark Parameters}

That an $n_{-}$-block is regarded as disordered mainly refers to magnetism, in the isomorph spin sense, however, it is also an ordered organization. So far, all thermodynamic parameters of the critical phenomena only involve the spin-interaction, ignoring the non-spin interaction. We call the parameters associated with the non-spin interaction such as energy, partition function, entropy, and free energy, 
etc. thermodynamic dark parameters, which affect the system's property as much as the conventional parameters do.

\section{Conclusion Remark}

It is first found that the non-Abelian gauge field is suitable for the non-spin electromagnetic interaction in the continue phase transition, in which the blocks illustrate Fermi-Bose duality. The gauge field maintains the self-similar transformation and makes system take the ordered path to minimum entropy reduction. The degeneracy of critical temperatures interprets the reason why the scaling law shows only nearby the first critical temperature for the real experimental specimens. The concept of thermodynamic dark parameters associated with the non-spin interaction is proposed firstly.

\section{Conflicts of Interest}

The author declares no conflicts of interest regarding the publication of this paper.

\section{References}

[1] Zinn-Justin, J. (2007) Phase Transitions and Renormalization Group. Oxford University Press, Oxford. https://doi.org/10.1093/acprof:oso/9780199227198.001.0001

[2] Moshe, M. and Zinn-Justin, J. (2003) Physics Reports, 385, 69-228. https://doi.org/10.1016/S0370-1573(03)00263-1

[3] Pelissetto, A. and Vicari, E. (2002) Physics Reports, 368, 549-727. https://doi.org/10.1016/S0370-1573(02)00219-3

[4] Feng, Y.G. (2018) Journal of Modern Physics, 9, 241-249.

[5] Weinberg, S. (1967) Physical Review Letters, 19, 1264. https://doi.org/10.1103/PhysRevLett.19.1264

[6] Yang, C.N. and Mills, R.L. (1954) Physical Review, 96, 191. https://doi.org/10.1103/PhysRev.96.191

[7] Bardeen, J., Cooper, L.N. and Schrieffer, J.R. (1957) Physical Review, 106, 162. https://doi.org/10.1103/PhysRev.106.162

[8] Goldstone, J., Salam, A. and Weinberg, S. (1962) Physical Review, 127, 965. https://doi.org/10.1103/PhysRev.127.965

[9] Feng, Y.G. (2018) Journal of Modern Physics, 9, 2035-2044. https://doi.org/10.4236/jmp.2018.911128

[10] Gray, R.M. (2009) Entropy and Information Theory. Springer-Verlag, New York.

[11] Binney, J.J., Dowrick, N.J., Fisher, A.J. and Newman, M.J. (1992) The Theory of Critical Phenomena: An Introduction to the Renormalization Group. Oxford University Press, Oxford.

[12] Reynolds, P.J., Stanley, H.E. and Klein, W. (1977) Journal of Physics C: Solid State Physics, 10, L167.

https://iopscience.iop.org/article/10.1088/0022-3719/10/8/002/meta

https://doi.org/10.1088/0022-3719/10/8/002 